\title{
Empresas multinacionales, derramas de conocimiento y spin-off en México. ¿El país de origen hace la diferencia?
}

\author{
Maximino Matus, ${ }^{*}$ Jorge Carrillo, ${ }^{* *}$ Redi Gomis***
}

Perfiles Latinoamericanos, 26(52) | 2018

DOI: $10.18504 / \mathrm{pl} 2652-007-2018$

Recibido: 31 de mayo de 2016

Aceptado: 11 de enero de 2018

\section{Resumen}

El artículo presenta un análisis acerca de las derramas de conocimiento por parte de las empresas multinacionales (EMN) en México y la generación de desprendimientos en relación con el origen de la inversión extranjera. Se realiza además un nuevo análisis de la encuesta "Firmas Multinacionales en México. Un estudio sobre la estructura organizacional, la innovación y las prácticas de empleo". El argumento que guía la exposición sugiere que dependiendo del nivel de centralización y formalización del modelo de negocios que distinguen a las EMN por país de origen, ellas darán lugar a desprendimientos con mayor o menor integración a las cadenas globales de valor (CGV).

\begin{abstract}
This article analyzes brain drain in multinational businesses (MNB's) in Mexico and the formation of spin-offs that result from foreign investment. We also offer a new analysis of findings from the "Multinational Firms in Mexico: A study of organizational structure, innovation, and employment practices" survey. We suggest that the level of centralization and the business model of each MNB is related to its country of origin, and that these factors may explain the emergence of spin-offs and the level of involvement these spin-offs have with global value chains.
\end{abstract}

Palabras clave: empresas multinacionales, desprendimiento empresarial, derrama de conocimiento, inversión extranjera directa, modelo de negocios, cadena global de valor.

Keywords: Multinational corporations, business detachment, spin-off knowledge, foreign direct investment, business, global value chains.

* Doctor en Sociología por la Universidad de Wageningen (Holanda). Investigador Conacyt adscrito al Departamento de Estudios Sociales, El Colegio de la Frontera Norte | matus@colef.mx.

** Doctor en Sociología por El Colegio de México. Investigador adscrito al Departamento de Estudios Sociales, El Colegio de la Frontera Norte | carrillo@colef.mx.

*** Doctor en Ciencias Sociales por El Colef (México). Investigador adscrito al Departamento de Estudios Sociales, El Colegio de la Frontera Norte | rgomis@colef.mx. 


\section{Introducción}

- a actividad de las empresas multinacionales (EMN) genera derramas de conocimiento en las regiones y países donde se establecen. Dichas derramas impactan positivamente en la economía local, ya que facilitan que otros actores entren en la actividad productiva asociada al giro de las multinacionales, y tienden a mejorar procesos de otras empresas o impulsar emprendimientos en otros sectores económicos. Cuando las empresas "desprendidas" logran insertarse de forma exitosa a las cadenas globales de valor (CGV) — como proveedores de las EMN-, o se consolidan como independientes, la economía local se beneficia. Sin embargo, pese a la evidencia sobre este impacto, existen pocos estudios que analicen a fondo cuáles son las características de las EMN que suelen generar derramas y promover desprendimientos, y menos aún se ha explorado a detalle la relación de dichos procesos con el país de origen de las EMN, en tanto este puede llegar a ser un elemento diferenciador de características importantes entre este tipo de empresas.

La hipótesis inicial de la investigación sugería que a mayor centralización y formalización en las operaciones de las EMN se incrementaría la probabilidad de la integración a las CGV por parte de las spin-offs originados, y viceversa. El análisis arrojó que la producción de desprendimientos es proporcional a la inversión de las EMN en la formación profesional de gerencias independientes, pero su integración a las CGV se relaciona con la centralización de las operaciones y ostracismo de la cultura empresarial; esto es, cuando el núcleo cultural y el control central de la EMN son férreos, el surgimiento de spin-off corporativos aumenta y, por el contrario, cuando el control es distribuido y las gerencias independientes, el surgimiento de spin-off empresariales se privilegia.

La exposición está dividida en seis secciones. Los dos primeros apartados revisan la literatura sobre la diversidad de derramas de conocimiento provenientes de las EMN y la consecuente formación de spin-offo desprendimientos, para enseguida discutir dos cuerpos de literatura que abordan el tema del efecto del país de origen (COE, por su sigla en inglés) y los sistemas nacionales de negocios (NBS, por su sigla en inglés). La tercera sección analiza el manejo de recursos humanos (HRM, por su sigla en inglés) por país de origen y formación de desprendimientos. La cuarta muestra los aspectos metodológicos relativos a los datos que sirvieron de base para el examen empírico. En la quinta sección se presentan los resultados del análisis de la encuesta "Firmas Multinacionales en México...” y los hallazgos generales. Por último, se exponen las conclusiones. 


\section{Derramas de conocimiento y desprendimientos}

La importancia de las spin-off tiene que ver en gran medida con el hecho de que sus protagonistas suelen ser beneficiarios de derramas de conocimiento. Independientemente de su tamaño, las empresas son repositorios de conocimiento. Como lo ha dicho Spencer (2008), cada organización posee su propia configuración y una de las capacidades centrales de las firmas recae precisamente en la gerencia y uso de este recurso intangible (Spencer, 2008: p. 345), el cual puede estar bien estructurado y sistematizado, o existir de forma tácita (Spencer, 2008; Monge-González, Leiva \& Alegre, 2011). El tamaño de la empresa puede estar relacionado con la cantidad de conocimiento que posee y gestiona; cuanto más grande, mayor sería su relación con dicho recurso, y viceversa. Sin embargo, esta relación no siempre se corresponde, ya que también existen pequeñas empresas intensivas en conocimiento como, por ejemplo, las que se especializan en el desarrollo y gestión de tecnologías de la información (TI), las cuales pueden operar en contextos locales o multinacionales, pues algunas "nacen globales" (Tanev, 2012).

Una relación tampoco correspondida, similar a la antes descrita, pero a la inversa, se expresa al interior de las EMN, las cuales por su tamaño no necesariamente son poseedoras de grandes cantidades de conocimiento, sino que ello dependerá del sector en el que se especialicen. Tal es el caso de las empresas multinacionales de alta tecnología (Емат) que, como lo han señalado Monge-González, Leiva \& Alegre (2011), por lo común poseen superioridad tecnológica y altas habilidades administrativas a nivel mundial. Estos mismos autores sostienen que, debido a esta superioridad, los países huéspedes de dichas empresas pueden obtener beneficios de su presencia mediante la transferencia y derrama de conocimiento que producen (Monge-González, Leiva \& Alegre, 2011: p. 9). ${ }^{1}$

Tanto la transferencia como la derrama de conocimiento pueden darse de manera formal y legal o informal y sin fundamento jurídico. El primer caso, esto es, la transferencia de conocimiento, se distingue por el establecimiento de acuerdos y alianzas reguladas jurídicamente entre la "empresa matriz" y la depositaria, esquema con el que ambas se benefician. La segunda posibilidad, es decir, la derrama de conocimiento, se refiere a la salida involuntaria de conocimiento de las

1 Görg (2007) ha señalado que la evidencia respecto de la productividad de la derrama tecnológica y de conocimiento no es contundente y considera que esto se debe a que "primero, las características individuales de cada país pueden influir hasta qué punto las derramas suceden, segundo, el nivel de capacidad de absorción de las firmas individuales es importante y tercero, enfocarse solamente en las derramas horizontales no toma en cuenta importantes canales verticales de transferencia de conocimiento, en particular a través de conexiones hacia atrás que pueden otorgar mayores efectos externos” (Görg, 2007: p. 240). 
EMN hacia una o varias empresas con las que no existen acuerdos jurídicos previos y por lo tanto ellas no obtienen beneficios directos (Javorcik, 2004).

Las derramas de conocimiento se dan por diversos medios. Monge-González, Leiva \& Alegre (2011) han identificado que, para las EMN establecidas en Costa Rica, ello sucede a través de tres vías: 1) demostración, 2) encadenamientos verticales y 3) movilidad laboral. Los mismos autores afirman que las del primer tipo incluyen la imitación, o ingeniería inversa por parte de empresas locales. Las segundas aluden a los eslabonamientos verticales, ya sea hacia delante o hacia atrás con las EMN, y el tercer caso se refiere a la transferencia y aplicación de conocimiento a empresas locales por parte de exempleados de las EMN (Monge-González, Leiva \& Alegre, 2011: p. 10).

Esas tres vías se mencionan con frecuencia en la literatura sobre las derramas (Altenburg, 2000; Fosfuri, Motta \& Rode, 2001; Spencer, 2008; De Fuentes \& Dutrénit, 2011); sin embargo, hay autores que han identificado otras variantes del proceso. Por ejemplo, Fosfuri, Motta \& Rode (2001) consideran que cuando las EMN pagan a los trabajadores un salario alto para prevenir que se muevan con un competidor local, también es posible hablar de derramas pecuniarias (Fosfuri, Motta \& Rode, 2001: p. 207). En tanto que Spencer (2008) señala que el "efecto de competencia" es un mecanismo que promueve las derramas, el cual hace referencia al aumento de la competitividad de la economía local por la presencia de las EMN. Empero, Spencer precisa que existe un efecto de competencia negativo dado que crece la presión por los recursos y entonces algunas empresas locales se ven imposibilitadas de seguir participando en el mercado (Spencer, 2008: p. 344). En tanto que Altenburg (2000) considera que los eslabonamientos horizontales que las EMN establecen con socios tecnológicos locales mediante joint ventures, acuerdos de licencia y alianzas estratégicas (Altenburg, 2000: p. 2), pueden calificarse de derrama.

Por su parte, De Fuentes \& Dutrénit, quienes hacia 2005 completaron un estudio sobre la capacidad de absorción de conocimiento de pymes mexicanas especializadas en maquinados industriales a partir de una encuesta levantada en el estado de Querétaro (De fuentes \& Dutrénit, 2007; De Fuentes, 2008), han propuesto que las "conexiones foráneas" que las pymes establecen para exportar a partir de la presencia de las grandes empresas en la región donde operan es otro tipo de derrama. Y, retomando a Görg \& Greenaway (2001), De Fuentes (2008) elabora una útil clasificación para distinguir los diferentes tipos de derrama, el origen de la ganancia productiva, la formalidad o informalidad del canal de difusión, y la naturaleza del eslabonamiento entre las grandes empresas y las pymes.

Desde nuestra perspectiva, algunas de las variantes identificadas por $\mathrm{Al}$ tenburg (2000), Fosfuri, Motta \& Rode (2001), Spencer (2008) y De Fuentes (2008), más que derramas, son mecanismos para transferencias o retención del 
conocimiento, ya que están reguladas legalmente o al menos tienen el potencial de ser perseguidas judicialmente. A partir de lo anterior, aquí se propone la existencia de tres principales motores en la promoción de las derramas y tres subtipos íntimamente ligados con los primeros, pero que por sus características se identifican más con mecanismos para la transferencia o retención del conocimiento. Es importante destacar que el proceso mediante el cual se promueven las derramas no limita su evolución a otro tipo o subtipo. Por ejemplo, una derrama informal horizontal iniciada por movilidad laboral es posible que con el tiempo genere un eslabonamiento formal vertical con la EMN de donde surgió, lo cual daría paso a una relación sin fundamento jurídico u otra regulada legalmente. En este tenor se propone el siguiente esquema para clasificar las derramas de conocimiento (cuadro 2).

Cuadro 1. Tipos de derrama, origen de la ganancia, canal de difusión y tipo de eslabonamiento

\begin{tabular}{|c|c|c|c|}
\hline Mecanismo & Origen de la ganancia productiva & $\begin{array}{l}\text { Canal de } \\
\text { difusión }\end{array}$ & $\begin{array}{l}\text { Tipo de } \\
\text { derrama }\end{array}$ \\
\hline 1. Eslabonamientos hacia atrás & $\begin{array}{l}\text { Conexiones de soporte } \\
\text { Incremento de eficiencia }\end{array}$ & Formal & Vertical \\
\hline 2. Movilidad de capital humano & $\begin{array}{l}\text { Incremento de productividad } \\
\text { Conocimiento tácito }\end{array}$ & Informal & $\begin{array}{l}\text { Vertical } \\
\text { Horizontal }\end{array}$ \\
\hline 3. Entrenamiento & Conocimiento tácito & Formal & Vertical \\
\hline 4. Transferencia directa de tecnología & Incremento en competitividad & Formal & Vertical \\
\hline 5. Demostración-imitación & $\begin{array}{l}\text { Adopción de nuevas metodologías productivas } \\
\text { Adopción de nuevas prácticas gerenciales }\end{array}$ & $\begin{array}{l}\text { Formal } \\
\text { Informal }\end{array}$ & $\begin{array}{l}\text { Vertical } \\
\text { Horizontal }\end{array}$ \\
\hline 6. Incremento de competencia & $\begin{array}{l}\text { Incremento de eficiencia } \\
\text { Adopción más rápida de nueva tecnología }\end{array}$ & $\begin{array}{l}\text { Formal } \\
\text { Informal }\end{array}$ & $\begin{array}{l}\text { Vertical } \\
\text { Horizontal }\end{array}$ \\
\hline 7. Eslabonamientos foráneos & $\begin{array}{l}\text { Economías de escala } \\
\text { Exposición internacional a mercados }\end{array}$ & $\begin{array}{l}\text { Formal } \\
\text { Informal }\end{array}$ & $\begin{array}{l}\text { Vertical } \\
\text { Horizontal }\end{array}$ \\
\hline
\end{tabular}

Fuente: Retomado de De Fuentes (2008). El cuadro es una adaptación de Görg \& Greenaway (2001).

\section{Cuadro 2. Tipos de derramas y sus características}

1. Derrama por eslabona- Empresas locales se insertan verticalmente en la cadena de la EMN. Por ejemplo, consmiento: vertical y formal truyendo relaciones hacia atrás como proveedores o hacia delante como distribuidores y marketing de productos.

1.1. Derrama por eslabona- Las EMN desarrollan proyectos conjuntos con empresas locales como socios, por ejemmiento: horizontal y formal plo en la forma de joint ventures, acuerdos de licencia o alianzas estratégicas.

2. Derrama por demostra- Empresas locales imitan o adaptan exitosamente las tecnologías y técnicas gerenciación: horizontal e informal les de las EMN.

2.1. Derrama por competen- Empresas locales aumentan su competitividad y aprenden a exportar debido a la precia: horizontal e informal sencia de EMN, pero también pueden ser eliminadas debido a que aumenta la presión sobre los recursos locales.

3. Derrama por movilidad Los trabajadores entrenados formalmente por la EMN o expuestos por largos periodos laboral: horizontal e a su ambiente laboral que son contratados por otras EMN, empresas locales o crean informal

sus propias empresas.

3.1. Derrama pecuniaria: Las EMN pagan más a los trabajadores locales con la finalidad de que no se vayan formal con la competencia o abandonen la empresa y con ello el conocimiento que poseen.

Fuente: Elaboración propia con base en Monge-González, Leiva \& Alegre (2011), Spencer (2008), Fosfuri, Motta \& Rode (2001) y Altenburg (2000). 
En este artículo hay especial interés por explorar las derramas del tercer tipo, es decir, las de movilidad laboral horizontal e informal. Al respecto, Altenburg (2000) afirma que por lo común las EMN invierten más en entrenamiento y, en consecuencia, los empleados desarrollan mayores capacidades en comparación con los que laboran en empresas locales, pero "inclusive, si los empleados de las TNC [EMN] no participan en actividades de entrenamiento, ellos pueden adquirir ciertas habilidades, actitudes e ideas solo por trabajar en una planta que está conformada por estándares internacionales de producción" (Altenburg, 2000: p. 4). Es decir, que a diferencia de los empleados que son entrenados formalmente por medio de procesos codificados, en el caso de los segundos, su exposición prolongada al nuevo ambiente laboral promueve que adquieran un conocimiento tácito. Para Gil \& Solís (2012), este último fenómeno se presume como un bien "tácito que permite al ex empleado de la EMN, tener ventajas tecnológicas y organizacionales” (Gil \& Solís, 2012: p. 667), tanto para crear una nueva empresa, como para competir con otras locales. La dispersión es posible gracias a lo intangible del conocimiento, de manera que no puede ser ni resguardado ni estar controlado por las multinacionales y fluye o se derrama de modo relativamente incontrolable fuera de ellas o de sus subsidiarias (Gil \& Solís, 2012: p. 667).

Diferentes autores han observado que cuando los conocimientos que poseen las EMN son altamente tácitos, la creación de empresas por parte de sus exempleados es la mejor forma de transferir ese conocimiento a las empresas locales (Spencer 2008; Monge-González, Leiva \& Alegre, 2011: p. 10). Esta creación de nuevas empresas se conoce como spin-off.

Parhankangas \& Arenius (2003) han conceptualizado los spin-off como "las nuevas formaciones de negocios basadas en las ideas de negocio desarrolladas dentro de la firma matriz que fueron tomadas por la firma constituida" (Parhankangas \& Arenius 2003: p. 464) y proponen distinguir los "spin-off corporativos", que fueron promovidos o permitidos por la firma matriz, de los "hostiles" o "competitivos" (Parhankangas \& Arenius 2003: p. 464), que, por el carácter informal de su origen, no son benéficos en primera instancia para la firma matriz. Por su parte, en estudios previos, Lindholm (1994) propuso que los spin-off corporativos podían clasificarse según el tipo de trasferencia de derechos de propiedad de la empresa matriz a su spin-off, y distinguió dos categorías: 1) spin-off enajenados: "la mayoría del poder de voto es transferido de una entidad existente legalmente a un nuevo cuerpo o una nueva firma" (Parhankangas \& Arenius, 2003: p. 464), y 2) spin-off empresarial: "por lo común no existe transferencia formal de derechos de propiedad. Más típicamente, un spin-offempresarial acurre cuando un empresario deja su empleo anterior para comenzar su propia firma" (Parhankangas \& Arenius, 2003: p. 464). Los del segundo tipo de Lindholm, es decir el empresarial, son los mismos que Parhankangas \& Arenius (2002: 
p. 464) identifican como hostiles o competitivos. ${ }^{2}$ Aquí se prefiere este término, y el de spin-off corporativo (Parhankangas \& Arenius, 2003) sobre el de enajenado (Lindholm, 1994).

\section{El efecto del país de origen y los sistemas nacionales de negocios}

Algunos autores han argumentado que independientemente de la intensificación de la globalización y las alianzas internacionales entre empresas provenientes de países diversos, las EMN conservan características distintivas de sus países de origen y de los modelos de negocios que practican (Ferner, 1997; Ferner \& Quintanilla, 1998; Sethi \& Elango, 1999; Almond, Edwards, Colling, Ferner, Gunnigle, Müller-Camen, Quintanilla \& Wüchter, 2005).

En la literatura sobre los rasgos que distinguen la forma de operar de las EMN de países diversos se han generado dos corrientes de pensamiento: 1) el efecto del país de origen (COE, por su sigla en inglés) y 2) los sistemas nacionales de negocios (NBS, por su sigla en inglés). En los siguientes párrafos describiremos brevemente estas perspectivas, para en seguida explicar de forma panorámica los modelos de negocios de los cuatro países y las dos regiones panétnicas analizadas en este estudio.

Ferner (1997) identifica varios problemas en los estudios que adoptan la perspectiva COE. Es en particular crítico de las generalizaciones hechas a partir de pocos estudios en profundidad donde "tradición" significa la identificación de algunos rasgos compartidos entre compañías de orígenes nacionales diversos. Por ejemplo, Ferner (1997) señala que con base en tres estudios de caso, uno holandés, otro anglo-holandés y uno sueco, Bartlett y Ghohal llegan a la conclusión de que "los modelos europeos dependen de la socialización a través del reclutamiento cuidadoso, desarrollo y aculturación de tomadores de decisiones clave" (Bartlett \& Ghohal, 1989: p. 163. Citado en Ferner, 1997: p. 22). Para Ferner, estas descripciones son tan amplias que prácticamente son válidas para cualquier EMN (Ferner, 1997: p. 22).

Otro problema identificado por Ferner (1997) en tales perspectivas, es su amplia dependencia respecto de la influencia que históricamente ha ejercido la expansión internacional del capital y su relación con la cultura nacional. También existe una explicación culturalista que enfoca la atención en el conocimiento

2 Se prefiere el concepto de Lindholm (1994) porque si bien los spin-off no regulados por las EMN pueden ser competitivos para con la misma, no necesariamente son hostiles, dado que en su desarrollo pueden forjar relaciones formales con la EMN de donde nacieron y aumentar así la competitividad de ambas empresas. 
de las diferencias nacionales en el estilo gerencial de la organización (Ferner, 1997: p. 23). Ferner considera que uno de los problemas metodológicos más profundos en estas perspectivas reside en la imposibilidad de distinguir qué tiene más peso para explicar las diferencias; esto es, ¿pesa más la cultura de origen o el estado de la organización?, ¿la "esencia" anglosajona, europea o asiática, o las fases de internacionalización, estructura corporativa, porción de operaciones fuera del país de origen, etc.? (Ferner, 1997: p. 23). Por lo anterior, este autor enfatiza en la importancia de distinguir los rasgos nacionales profundos - que afectan el comportamiento de las EMN - de los contingentes (Ferner, 1997: p. 24). Y sugiere considerar que los efectos de la acelerada modernización sobre "las instituciones políticas, el rápido paso del cambio tecnológico, la internacionalización de la producción en sí misma, y los patrones cambiantes de los mercados internacionales y la competencia, son capaces de modificar las estructuras nacionales pre-existentes (Ferner, 1997: p. 24).

La segunda corriente de pensamiento que aborda el tema de los rasgos de la forma de operar de las EMN de países diversos es la del NBs. Al respecto, Ferner (1997) argumenta que en esta perspectiva se encuentran dos grandes cuerpos de literatura. Por un lado, la que desde un punto de vista institucional o estructuralista argumenta que los diferentes elementos del NBs "se interrelacionan en un todo complejo, dando impulso a patrones característicos de comportamiento de negocios en países diferentes que persisten en el tiempo" (Ferner, 1997: p. 24); y que explora temas como la "gobernanza corporativa, gerencia de estructuras y división funcional del trabajo, IR [relaciones industriales] e instituciones laborales del mercado, sistemas de entrenamiento, organización del lugar de trabajo, etc." (Ferner, 1997: p. 24).

La segunda aproximación es de carácter culturalista. Ferner advierte que uno de sus principales exponentes es Hofstede (1980), quien ha explorado las "percepciones individuales de poder y autoridad, orientaciones al individualismo y la acción colectiva, y actitudes a corto y largo plazo" (Ferner, 1997: p. 25). En ambas aproximaciones la pregunta clave es "hasta qué punto dichas diferencias culturales en los sistemas de negocios orientan el comportamiento de las EMN en los diferentes países" (Ferner, 1997: p. 25). Crítico de ambas posturas, Ferner argumenta que algunas características de los NBs simplemente no son factibles en un contexto de la globalización intensiva, ya que dichas propuestas están aisladas de la constelación de elementos que les dan sentido (Ferner, 1997: p. 25). En cambio, afirma que pueden existir diferentes grados de isomorfismo, dependiendo de la clase de arreglos institucionales a los que las EMN lleguen con los países donde operan (Ferner \& Quintanilla, 1998).

Como alternativa a las corrientes de pensamiento mencionadas, Ferner \& Quintanilla (1998) argumentan que los NBS pueden verse como "clúster 
de instituciones inter-ligadas y elementos de culturas de negocios" (Ferner \& Quintanilla, 1998: p. 715). No obstante, estos autores observan que estas influencias no son deterministas "pues los NBs son en sí mismos heterogéneos y plurales (Mayer \& Whittington, 1996), con variables considerables" (Ferner \& Quintanilla, 1998: p. 715).

Por su parte, al analizar la operación de EMN en varios países europeos, Almond et al., (2005) argumentan que las EMN tienen lazos con múltiples países "no solo el sistema del países de donde se origina, sino también de las sociedades donde se hospeda y opera" (Almond et al., 2005: p. 278), lo cual afecta las operaciones de la corporación en numerosas direcciones. Por ello, para explicar la complejidad de las EMN sugieren que es necesario emprender un análisis institucional multinivel donde "cada grupo de efectos potenciales varía a través del tiempo y espacio, tanto en respuesta a la economía externa y el cambio macro-institucional, como a las sub-micro estrategias adoptadas por los actores a varios niveles de la organización" (Almond et al., 2005: p. 281). Desde nuestra perspectiva, la aproximación propuesta por estos autores es muy sugerente, pero sumamente complicada de implementar para llegar a conclusiones específicas que comprendan sistémicamente al total de las relaciones institucionales y los múltiples niveles que afectan el desempeño de las EMN en un espacio y momento determinado. Por ello, en este artículo nos limitamos a revisar a algunas áreas del manejo de recursos humanos que, consideramos, afectan positiva o negativamente la promoción de spin-off empresariales o corporativos por parte de las EMN con orígenes compartidos.

\section{Manejo de recursos humanos por país de origen y generación de spin-off}

En esta sección ofrecemos una sucinta revisión de la literatura sobre la forma como las EMN manejan su área de recursos humanos según su región o país de origen. Los modelos son Alemania, Francia, Japón y China, más dos regionales o panétnicos: la tradición anglosajona y la europea continental.

a) El modelo anglosajón.

En primer lugar es posible diferenciar las corporaciones estadounidenses de las inglesas porque las primeras aplican de forma más intensiva el sistema de administración anglosajón que, en términos resumidos, se puede caracterizar como centralizado/vertical y formal con procesos de operación bien documentados y donde se promueve el desempeño mediante el uso cotidiano de un sistema de recompensas (Hampden-Turner \& Trompenaars, 1993; Lawrence, 1996b; Esterby-Smith et al., 1995. Citados en Yan, 2003: pp. 490-491). 
En cuanto a la relación del sistema de HRM anglosajón y la promoción o inhibición del proceso de spin-off, identificamos que debido a su administración central, formal y racionalista, tanto en el manejo de los recursos humanos, en general, como en la formación y desarrollo de gerentes en particular, la transferencia de conocimiento y la movilidad del personal internacional se facilitan. Sin embargo, el surgimiento de spin-off empresariales (Lindholm, 1994) se inhibe, pues forman pocos cuadros locales y se inclinan por la promoción de spin-off corporativos (Parhankangas \& Arenius, 2003), regulados por un marco jurídico donde domina la lógica del contrato sobre las relaciones de confianza.

b) El modelo europeo.

El modo de administración de las EMN europeas puede caracterizarse como un sistema que se basa en una ingeniería de procesos altamente formalizados y estandarizados pero con un control de mando informal, ya que este recae fuertemente en la supervisión personal de un gerente experimentado (Ebster-Grosz \& Pugh, 1996. Citado en Yan, 2003: p. 491). Sin embargo, las subsidiarias no siempre adoptan las políticas y prácticas estandarizadas de la organización central y sus gerentes gozan de cierta autonomía, lo cual es propicio para originar spin-off empresariales, pues como lo dicen Contreras \& Carrillo (2011: p. 345), "la existencia de una mayor autonomía en la toma de decisiones [...] favorece el surgimiento de iniciativas empresariales". Por otra parte, la baja centralización de las subsidiarias podría estar promoviendo la integración de los spin-off empresariales a las CGV de las EMN que los generaron (Forsgren, 1990. Citado en Yan, 2003: p. 491). Y el hecho de que la cultura empresarial europea recaiga con mayor grado en la lealtad y la confianza puede ser otro aliciente para la promoción de spin-offempresariales, ya que el término de este tipo de relaciones no es perseguido jurídicamente.

c) El modelo alemán y francés.

Al comparar el modelo de las EMN europeas con el caso específico del alemán y francés, surgen similitudes y diferencias importantes. Mientras el modelo alemán concibe a todos los empleados como valiosos para la compañía y busca crear una conciencia colectiva basada en la confianza y la lealtad, el francés promueve la individualidad y discrecionalidad. Asimismo, en el alemán queda claro que su alta centralización y dependencia respecto de los gerentes expatriados (Ferner \& Quintanilla, 1998: p. 716) inhibe los spin-offempresariales promovidos por extrabajadores en puestos gerenciales. Entre tanto, la alta jerarquía que distingue al modelo francés donde la información relevante suele concentrarse en individuos clave conduce a interfaces de conocimiento (Long, 
2007) que dificultan la integración de las subsidiarias, e inhibe las derramas de conocimiento, hecho que podría reducir a su vez la generación de spin-off empresariales por parte de gerentes locales.

En su análisis del caso alemán y francés, Ferner \& Quintanilla (1998) señalan que ambas naciones han ido adaptando sus modelos de negocio desde hace un par de décadas. Por ejemplo, en el alemán "han introducido divisiones internacionales de productos con una estrategia descentralizada de responsabilidades" (Ferner \& Quintanilla 1998: p. 19) con lo que se busca flexibilidad y capacidad responsiva de parte de los gerentes. Algunas EMN también han adoptado un modelo de incentivos en relación al actuar de las gerencias y el valor del accionista se ha resaltado (Ferner \& Quintanilla 1998: p. 719). El modelo francés ha hecho lo propio, particularmente en lo tocante a la introducción de divisiones internacionales con una estrategia descentralizada de responsabilidades (Ferner \& Quintanilla, 1998: p. 719). Por ello Ferner y Quintanilla sugieren que Alemania y Francia marchan hacia el modelo anglosajón a fin de adaptar sus operaciones a la competencia internacional, lo cual no implica que se borren algunos de los rasgos que distinguen a sus sistemas de negocios nacionales (Ferner $\&$ Quintanilla, 1998), sino que más bien, retomando un concepto del antropólogo estructuralista Lévi-Strauss (1966), nos encontramos frente a un fenómeno de "bricolaje", donde se utilizan "los elementos disponibles para construir artefactos culturales muy diferentes” (Ferner \& Varul, 2000: p. 137).

\section{d) El modelo japonés.}

El modelo japonés de administración de las EMN puede ser definido como centralizado, informal y jerárquico, donde las políticas de reclutamiento, selección y entrenamiento son compartidas por las subsidiarias. Los empleados son considerados miembros de la familia empresarial y las relaciones que establecen con estos y el resto de los actores de la cadena suelen ser sustentables y de larga duración. Se piensa que debido a la dependencia y lealtad que los gerentes guardan a la corporación, el surgimiento no regulado de spin-off empresariales es limitado. Lo que no sucede con la fuerza laboral que forman en los países de hospedaje, pues estos reciben entrenamiento continuo que después pueden utilizar para iniciar sus emprendimientos. Empero, a causa de que es una cultura afectiva y centrada en el colectivo, se sostiene que los spin-off que fundan empleados foráneos u autóctonos, difícilmente se podrán integrar vertical u horizontalmente a las CGV de las EMN que las originaron, ya que estos emprendimientos serían sancionados moralmente. Otro factor que limita la formación de spin-offempresariales en el modelo japonés es su alta dependencia de los gerentes expatriados quienes concentran el poder y conocimiento respecto a la administración y reciben amplios incentivos para resguardarlo. 
e) El modelo chino.

El modelo chino de administración de las EMN puede definirse como centralizado, informal y jerárquico, además de estar fuertemente basado en la familia y los lazos de confianza y lealtad. En sus prácticas de reclutamiento prestan especial atención a la cercanía social de los empleados y gerentes, más que a su competitividad. Si bien forman gerentes dependientes, destaca su capacidad para crear spin-off del tipo corporativo, los cuales son administrados por miembros de la familia o socios cercanos socialmente. Esto asegura la unión de las filiales asociadas. Por el amplio énfasis que ponen en la confianza y la lealtad, y por ser un modelo altamente jerarquizado, resulta poco factible la formación de spin-off empresariales.

Cuadro 3. Tradiciones nacionales en la administración y gerencia de las EMN. Modelos anglosajón, europeo continental, francés, alemán, japonés y chino

\begin{tabular}{|c|c|c|c|c|c|c|c|}
\hline & 1. HRM & $\begin{array}{l}\text { 2. Política } \\
\text { laboral }\end{array}$ & $\begin{array}{l}\text { 3. Formación } \\
\text { de gerentes }\end{array}$ & $\begin{array}{l}\text { 4. Desarrollo } \\
\text { de gerentes }\end{array}$ & $\begin{array}{l}\text { 5. Cultura } \\
\text { empresarial }\end{array}$ & $\begin{array}{l}\text { 6. Foco de } \\
\text { atención en la } \\
\text { cadena }\end{array}$ & $\begin{array}{l}\text { 7. Relación con } \\
\text { proveedores } \\
\text { e instituciones } \\
\text { financieras }\end{array}$ \\
\hline $\begin{array}{l}\text { I. Anglosa- } \\
\text { jona }\end{array}$ & $\begin{array}{l}\text { Centralizada } \\
\text { y formal }\end{array}$ & $\begin{array}{l}\text { Centralizada } \\
\text { y formal }\end{array}$ & $\begin{array}{l}\text { Homogeniza } \\
\text { y formal }\end{array}$ & Autónomos & $\begin{array}{l}\text { Racional y } \\
\text { centrada en } \\
\text { el individuo }\end{array}$ & Finanzas & $\begin{array}{l}\text { Cortoplacista, } \\
\text { orientada por } \\
\text { el mercado }\end{array}$ \\
\hline $\begin{array}{l}\text { II. Europea } \\
\text { continental }\end{array}$ & $\begin{array}{l}\text { Descen- } \\
\text { tralizada y } \\
\text { formal }\end{array}$ & $\begin{array}{l}\text { Descen- } \\
\text { tralizada y } \\
\text { formal }\end{array}$ & $\begin{array}{l}\text { Heterogénea } \\
\text { y formal }\end{array}$ & $\begin{array}{l}\text { Semidepen- } \\
\text { dientes }\end{array}$ & $\begin{array}{l}\text { Afectiva y } \\
\text { centrada en } \\
\text { el individuo }\end{array}$ & $\begin{array}{l}\text { Producción y } \\
\text { comercial }\end{array}$ & $\begin{array}{l}\text { Largo plazo e } \\
\text { intervencionista }\end{array}$ \\
\hline III. Alemania & $\begin{array}{l}\text { Centralizada } \\
\text { y formal }\end{array}$ & $\begin{array}{l}\text { Centralizada } \\
\text { y formal }\end{array}$ & $\begin{array}{l}\text { Heterogénea } \\
\text { y formal }\end{array}$ & Dependientes & $\begin{array}{l}\text { Racional y } \\
\text { entrada en el } \\
\text { colectivo }\end{array}$ & $\begin{array}{l}\text { Producción y } \\
\text { comercialización }\end{array}$ & $\begin{array}{l}\text { Largo plazo e } \\
\text { intervencionista }\end{array}$ \\
\hline IV. Francia & $\begin{array}{l}\text { Centralizada } \\
\text { y formal }\end{array}$ & $\begin{array}{l}\text { Centralizada } \\
\text { y formal }\end{array}$ & $\begin{array}{l}\text { Heterogénea } \\
\text { y formal }\end{array}$ & Dependientes & $\begin{array}{l}\text { Afectiva y } \\
\text { centrada en } \\
\text { el individuo }\end{array}$ & $\begin{array}{l}\text { Producción y } \\
\text { comercialización }\end{array}$ & Sin información \\
\hline V. Japón & $\begin{array}{l}\text { Centralizada } \\
\text { e informal }\end{array}$ & $\begin{array}{l}\text { Centralizada } \\
\text { e informal }\end{array}$ & $\begin{array}{l}\text { Heterogénea } \\
\text { e informal }\end{array}$ & Dependientes & $\begin{array}{l}\text { Afectiva y } \\
\text { centrada en } \\
\text { el colectivo }\end{array}$ & $\begin{array}{l}\text { Producción y } \\
\text { comercialización }\end{array}$ & $\begin{array}{l}\text { Largo plazo e } \\
\text { intervencionista }\end{array}$ \\
\hline VI. China & $\begin{array}{l}\text { Centralizada } \\
\text { e informal }\end{array}$ & $\begin{array}{l}\text { Centralizada } \\
\text { e informal }\end{array}$ & $\begin{array}{l}\text { Heterogénea } \\
\text { e informal }\end{array}$ & Dependientes & $\begin{array}{l}\text { Afectiva y } \\
\text { centrada en } \\
\text { el colectivo }\end{array}$ & $\begin{array}{l}\text { Producción y } \\
\text { comercialización }\end{array}$ & $\begin{array}{l}\text { Largo plazo e } \\
\text { intervencionista }\end{array}$ \\
\hline
\end{tabular}

Fuente: Elaboración propia con base en Ferner (1997), Ferner \& Quintanilla (1998) y Yan (2003).

Resumiendo este apartado, a partir de la descripción y clasificación de los modelos de administración de negocios las EMN que distinguen a los cuatro países y dos regiones panétnicas analizadas (cuadro 3), es posible llegar a las siguientes reflexiones acerca del impacto que tienen las tipologías presentadas sobre la naturaleza de los desprendimientos:

a) Modelo anglosajón: la generación de spin-off se encuentra regulada por un paradigma de eslabonamiento vertical y formal regulado jurídicamente. 
Dichas condiciones privilegian la creación de spin-off corporativos e inhiben la de spin-off empresariales en el mismo giro.

b) Modelo europeo: la generación de spin-offse encuentra escasamente regulada jurídicamente, lo cual, sumado a la cultura afectiva (lealtad y confianza) que caracteriza sus relaciones, promueve las condiciones propicias para la formación de spin-off empresariales.

- Modelo alemán: la fundación de spin-off empresariales por parte de gerentes se inhibe por la alta centralización y dependencia respecto de los gerentes expatriados. Pero además, el reconocimiento como socios y las amplias prestaciones que se dan a los trabajadores de las EMN alemanas podrían, de igual modo, estar frenando el surgimiento de spinoff de puestos no gerenciales.

- Modelo francés: la generación de spin-off empresariales por parte de puestos gerenciales se inhibe por su modelo de alta jerarquía, ya que el conocimiento relevante suele concentrarse en individuos clave para la organización, hecho que dificulta las derramas de conocimiento.

c) Modelo japonés: la generación de spin-off se encuentra escasamente regulada jurídicamente, pero debido a lo estricto de su cultura afectiva (lealtad y confianza) y colectiva, la promoción de spin-offempresariales queda inhibida al sancionarse moralmente, por ello los de tipo corporativo son privilegiados.

d) Modelo chino: la generación de spin-off no se encuentra regulada jurídicamente, difícilmente surgirán desprendimientos corporativos por la jerarquía del grupo social. Igualmente, debido al ostracismo de su cultura afectiva (lealtad y confianza) basada en el colectivo cercano socialmente, la promoción de spin-off empresariales prácticamente es imposible.

\section{Los datos de la encuesta}

La información que servirá de fuente primaria para esta sección del trabajo proviene de una encuesta efectuada a una muestra de 171 firmas multinacionales en México —en un universo de 922-, entre fines de 2008 y principios de 2009. ${ }^{3}$ La encuesta se produjo en el marco de un proyecto de investigación desarrollado en El Colegio de la Frontera Norte, con el objetivo de estudiar la

3 Aunque se diseñó una muestral aleatoria estratificada (por origen, sector y tamaño), por diferentes razones contextuales (crisis mundial, violencia en el país, epidemia AH1N1), resultó alta la tasa de no respuesta. Esto afecta la representatividad de la muestral y, en consecuencia, la generalización de las conclusiones. 
estructura organizacional, la innovación y las prácticas de empleo de las empresas multinacionales en México. Este proyecto, a su vez, siguió de cerca los lineamientos establecidos por Intrepid, ${ }^{4}$ una red internacional de investigadores independientes que busca consolidar, con el aporte de todos los grupos nacionales participantes, una base de datos confiable y relativamente homogénea que lleve a producir análisis comparables de alta calidad sobre políticas y prácticas de empleo de las empresas multinacionales alrededor del mundo.

La población objetivo de la investigación resulta de la definición operativa adoptada de común acuerdo por los participantes en Intrepid, según la cual se considerará como multinacionales a aquellas firmas que tienen actividad económica en por lo menos otro país además de México, con al menos un total de quinientos empleados, y un mínimo de cien en el país donde se origina la encuesta. $^{5}$

\section{Análisis de los datos de la encuesta y hallazgos generales}

Antes de pasar al examen concreto de los desprendimientos empresariales a partir de los datos de la encuesta, es importante ofrecer un breve perfil de las empresas que respondieron el cuestionario, en algunos aspectos. ${ }^{6}$ Así, en términos del origen del capital, en México se encuentran inversiones de casi todos los continentes. El 87\% de las firmas encuestadas fueron extranjeras. De estas últimas, las inversiones de Estados Unidos fueron las principales, aunque resultaron significativas las procedentes de Japón, Alemania y Francia, entre otros. En cuanto al sector, el $78.4 \%$ desarrolla su actividad en la industria, sobre todo en la manufacturera, mientras que el $21.6 \%$ pertenece a los servicios.

En seguida se presenta el análisis de los datos de la encuesta directamente relacionados con el fenómeno en examen.

$4 \quad$ Intrepid es el acrónimo de Investigation of Transnational Employment Practices: An International Database. En esta red participan investigadores de Inglaterra, Irlanda, España, Canadá, México, Argentina, Noruega, Finlandia y Australia. Sus miembros se reúnen aproximadamente cada dos años para intercambiar información y trazar estrategias de investigación de largo aliento, enfatizando en los estudios comparados.

5 Se insiste en que los objetivos del proyecto para el cual fue levantada la encuesta no iban dirigidos expresamente al estudio de los desprendimientos empresariales de las EMN. Pero aunque por esa razón hay limitaciones para un examen muy profundo de la problemática, los datos de la encuesta permiten fundamentar algunos análisis al respecto, y avanzar en la comprensión del fenómeno, en específico en cuanto a la impronta que en su manifestación tiene el origen nacional de las inversiones.

6 Para un perfil más completo de las EMN de la encuesta, puede revisarse Carrillo \& Gomis (2011). 
En al cuadro 4 se aprecia que la mayor parte de las spin-offs de alguna manera vinculadas a las EMN se originaron en empresas aquí categorizadas como anglosajonas. Prácticamente tres cuartas partes de los desprendimientos $(72.2 \%)$ corresponden a EMN de este origen, en tanto que una décima parte pertenece a las europeas y aproximadamente un quinto $(17.8 \%)$ son japonesas. Pudiera pensarse que estas proporciones guardan relación directa con el volumen de las EMN en cada una de estos tres tipos considerados. Y, en efecto, el grupo anglosajón es el de mayor peso. Sin embargo, la explicación va más allá de esto. Mientras en este grupo se dispara el peso de las spin-offs, de 60 a $72 \%$, este se reduce en los grupos de Europa y Asia. Y esto ocurre porque, en promedio, a nivel de empresas, las anglosajonas tienen más desprendimientos que las europeas (5.8 vs. 4.6), y casi el doble de las asiáticas (5.8 vs. 3).

Los datos de la encuesta corroboran, al menos en parte, que el impulso para fomentar desprendimientos en las EMN se relaciona con los esfuerzos por favorecer el desarrollo de los gerentes. De acuerdo a esos datos, las empresas anglosajonas y europeas son justamente las que más usan mecanismos de desarrollo gerencial para desarrollar "altas potencialidades" o "potenciar a los gerentes".

Cuadro 4. Información sobre spin-offs de EMN en México, por origen del capital

\begin{tabular}{lcccc}
\hline Origen & $\begin{array}{c}\text { Porcentaje (\%) } \\
\text { de N }\end{array}$ & $\begin{array}{c}\text { Total de spin-offs } \\
\text { en EMN }\end{array}$ & $\begin{array}{c}\text { Porcentaje (\%) de } \\
\text { spin-offs }\end{array}$ & $\begin{array}{c}\text { Promedio de spin- } \\
\text { offs por empresa }\end{array}$ \\
\hline Anglosajón (EE. UU. y U. K.) & 60 & 626 & 72.20 & 5.8 \\
Europa & 11 & 87 & 10.00 & 4.6 \\
Asia & 29 & 154 & 17.80 & 3 \\
Total & 100 & 867 & 100.00 & 4.9 \\
\hline
\end{tabular}

Fuente: Encuesta de Corporaciones Multinacionales. Proyecto COLEF-CONACYT \#55108, "Firmas Multinacionales en México. Un estudio sobre la estructura organizacional, la innovación y las prácticas de empleo”, 2008-2009.

Si bien el cuadro 4 sugiere que las EMN anglosajonas son las que más spin-off producen en el país, tanto a nivel global — total de la muestra- como individual —empresas-, al desagregar la muestra por el tipo de desprendimiento generado, es decir, empresarial vs. corporativa, se halla que la relación se modifica significativamente (cuadro 5).

En efecto, aunque las anglosajonas dan lugar en promedio a prácticamente el doble de spin-off (5.8) que las asiáticas (3.0), las primeras son menos dadas a apoyar corporativamente a los gerentes emprendedores (16.8\%); en cambio, las últimas favorecen más estos spin-off(30.8\%); entre las europeas la formación de este tipo de desprendimientos es casi nulo (7.4\%). Se sobreentiende que el resto 
de los desprendimientos son de tipo empresarial, es decir, que no son arropados por los corporativos y, por tanto, no se incorporan a las CGV de las EMN que les dieron origen: $69.2 \%$ asiáticas, $83.2 \%$ anglosajonas y $92.6 \%$ europeas.

Cuadro 5. EMN en México con spin-offs proveedores de la misma, por origen del capital

\begin{tabular}{lccc}
\hline Origen & Sí(\%) & No (\%) & Total (\%) \\
\hline Anglosajón (EE. UU. y U. K.) & 16.80 & 83.20 & 100 \\
Europa & 7.40 & 92.60 & 100 \\
Asia & 30.80 & 69.20 & 100 \\
Total & 19.40 & 80.60 & 100 \\
\hline
\end{tabular}

Fuente: Encuesta de Corporaciones Multinacionales. Proyecto COLEF-CONACYT \#55108, "Firmas Multinacionales en México. Un estudio sobre la estructura organizacional, la innovación y las prácticas de empleo", 2008-2009.

Estos resultados parecen contradecir a primera vista la hipótesis original de que a mayor centralización y formalización en las operaciones de las EMN, se incrementaría la integración a las CGV por parte de los spin-offs formados, y viceversa. En contraste con la revisión teórica, las empresas anglosajonas y europeas son las que más desprendimientos producen, pero las que menos integran a los spin-off a sus CGV. De manera opuesta, las empresas asiáticas son las que menos desprendimientos producen, pero su integración a las CGV es mayor.

Esta apreciación es congruente con la revisión teórica respecto de la impronta del origen sobre la organización y prácticas que siguen las filiales de las EMN, ya descritas páginas arriba. No obstante, vale aclarar que ese cuerpo de literatura examina en gran medida el comportamiento de EMN en países desarrollados de Europa. Cabría preguntarse entonces acerca de si ese tipo de empresas opera de forma semejante en México.

El factor autonomía en la cadena de valor

Con el propósito de explorar cómo se conecta este factor con el fenómeno examinado, se creó una variable que indica el grado de autonomía para tomar decisiones operativas con que gozan las EMN encuestadas en relación con sus matrices. Para facilitar el análisis se dicotomizó la variable en cuestión. El cruce de esta con los mismos indicadores usados en el cuadro 4 para examinar el impacto del origen de capital, se resumen en el cuadro 6.

Opuestamente a lo previsible, estos datos no son incompatibles con la hipótesis de partida, como parecía desprenderse de los análisis previos. Los niveles bajos de autonomía, que pueden tomarse como equivalentes de niveles altos de 
centralidad, son clave para entender el fenómeno de los desprendimientos en los aspectos que han sido hasta ahora examinados. Esto es: generan 2.4 veces más spin-offs que las empresas menos centralizadas; producen en promedio casi el doble de desprendimientos (6.2 vs. 3.4), y los mismos tienden a ser sobre todo del tipo corporativo, integrados a las CGV ( $80 \%$ vs. $20 \%)$.

Cuadro 6. Información sobre spin-offs de EMN en México, por nivel de autonomía en la cadena de valor de las empresas

\begin{tabular}{lcccc}
\hline $\begin{array}{l}\text { Nivel de } \\
\text { autonomía }\end{array}$ & EMN con spin-offs (\%) & $\begin{array}{c}\text { Total de spin-offs en } \\
\text { EMN }\end{array}$ & $\begin{array}{c}\text { Promedio de spin-offs } \\
\text { por EMN }\end{array}$ & $\begin{array}{c}\text { Spin-offs corporativas } \\
\text { (\%) }\end{array}$ \\
\hline Bajo & 55.5 & 713 & 6.2 & 80.0 \\
Alto & 44.5 & 293 & 3.4 & 20.0 \\
Total & 100 & 1006 & 5.0 & 100 \\
\hline
\end{tabular}

Fuente: Encuesta de Corporaciones Multinacionales. Proyecto COLEF-CONACYT \#55108, "Firmas Multinacionales en México. Un estudio sobre la estructura organizacional, la innovación y las prácticas de empleo", 2008-2009.

¿Dónde se produce, entonces, la discrepancia advertida? Como fue sugerido más arriba, la organización y comportamiento de las EMN en los países subdesarrollados es posible que no tenga el mismo trazo que el desplegado dentro de las sociedades del primer mundo. Como se aprecia en el cuadro 7 , contrario a lo reflejado por la literatura, para el caso mexicano las EMN de origen anglosajón resultan ser las menos centralizadas y jerárquicas, rasgos sobresalientes en las de origen europeo. La autonomía en sus decisiones es baja en el 55\% de las anglosajonas, en el 64\% de las europeas y en el 60\% de las asiáticas. Esto es un hallazgo que amerita análisis posteriores. Baste por ahora señalar que, sin desestimar los puntos de vista que atribuyen un carácter determinante al efecto derivado del origen nacional de las empresas, es necesario reconocer la importancia de otros factores. Y que siempre es imprescindible contrastar empíricamente antes de aceptar como válidas las conclusiones emanadas de estos en contextos de desarrollo con diferencias importantes.

Cuadro 7. Nivel de autonomía en la cadena de valor de las EMN en México por origen del capital

\begin{tabular}{lccc}
\hline \multirow{2}{*}{ Origen } & \multicolumn{2}{c}{ Nivel de autonomía } & Total (\%) \\
\cline { 2 - 3 } & Bajo (\%) & Alto (\%) & 100 \\
\hline Anglosajón (EE. UU. y U. K.) & 54.6 & 45.4 & 100 \\
Europa & 64.1 & 35.9 & 100 \\
Asia & 59.9 & 40.1 & 100 \\
Total & 59.9 & 40.1 & 100 \\
\hline
\end{tabular}

Fuente: Encuesta de Corporaciones Multinacionales. Proyecto COLEF-CONACYT \#55108, "Firmas Multinacionales en México. Un estudio sobre la estructura organizacional, la innovación y las prácticas de empleo", 2008-2009. 


\section{Conclusiones}

A partir de la discusión y los datos presentados se concluyen las siguientes ideas:

— Es obvia la influencia del país de origen sobre algunos atributos de los desprendimientos. Por mucho, son las EMN anglosajonas en las que se originan con mayor fuerza los procesos de spin-offs. Entre las EMN que operan en México el volumen de los desprendimientos se relaciona con la extensión con la que se usan mecanismos para potenciar las capacidades de los gerentes. Una cualidad más afín a los modelos anglosajón y europeo, y menos en los asiáticos. Un resultado congruente con lo recogido por la literatura global sobre el tema.

- Sin embargo, la influencia del origen se altera respecto de la idea anterior si en vez del volumen de desprendimientos se observa el tipo de spin-offs. Aquí son las asiáticas las que más influyen en la formación de desprendimientos corporativos y no las anglosajonas o europeas. Esto es relevante porque estas nuevas empresas son integradas a las CGV. El elemento de más trascendencia en términos explicativos parece recaer sobre la centralización de las operaciones y ostracismo de la cultura empresarial, y en donde a las filiales se les restringen los grados de libertad para tomar decisiones sobre las operaciones. Este rasgo es más frecuente en México cuando se trata del modelo asiático. En sentido opuesto, cuando el control es distribuido y las gerencias gozan de mayor independencia, se privilegia la generación de spin-off empresariales, lo cual aparece con mayor fuerza entre las EMN que operan en México y en los modelos anglosajón y europeo. De acuerdo a la literatura revisada, es un resultado que modifica lo esperado en términos de la relación entre origen y autonomía. Evidencia, una vez más, la necesidad de contrastar empíricamente las ideas derivadas de otros contextos, y no asumirlas e incorporarlas acríticamente.

Se puede concluir que el origen del capital es un factor importante para explicar las características asumidas por el fenómeno de los desprendimientos de nuevas empresas gestadas desde las EMN que operan en México. Sin embargo, por un lado, no existen los elementos suficientes para sostener que sea el único factor de peso. Tampoco, por otra, que expliquen por sí solos los mecanismos mediante los cuales se ejerce esa influencia.

Finalmente, cabe subrayar que las ideas presentadas invitan a la reflexión sobre qué tipo de iniciativas y políticas públicas necesitamos para promover desprendimientos que beneficien a la economía y a los procesos de innovación del país. ¿Es preferible tener un número significativo de spin-off empresariales 
que no se integran a las CGV y desaparecen rápidamente por no seguir incursionando en el giro de la EMN que los produjo, o conviene alentar desprendimientos corporativos que se incorporan de forma exitosa a las CGV? ¿Cuál sería el equilibrio adecuado de esta relación?

\section{Referencias}

Almond, P., Edwards, T., Colling, T., Ferner, A., Gunnigle, P., Müller-Camen, M., Quintanilla, J. \& Wächter, H. (2005). Unraveling Home and Host Country Effects: An Investigation of de HR Policies of an American Multinational in Four European Countries. Industrial Relations, 44(2), 276-306.

Altenburg, T. (2000). Linkages and Spill-overs between Transnational Corporations and Small Medium-Sized Enterprises in Developing Countries. Opportunities and Policies. Reports and Working Papers núm. 5, Berlín: German Development Institute.

Carrillo, J. \& Gomis, R. (2011). Un estudio sobre prácticas de empleo en firmas multinacionales en México: Un primer mapeo. Frontera Norte, 23(46), 35-60.

Contreras, O. \& Carrillo, J. (2011). Las empresas multinacionales como vehículos para el aprendizaje y la innovación de las empresas locales. En Bracamonte, Á. \& Contreras, O. F. (Eds.). Ciencia, tecnología e innovación para el desarrollo económico (pp. 325-354). Hermosillo, Sonora, México: El Colegio de Sonora/Conacyt.

De Fuentes, C. (2008). Different patterns of Knowledge spillovers mechanism in two Mexican localities. Documento presentado en la IV Globelics Conferencia at Mexico City, 22-24 de septiembre.

De Fuentes, C. \& Dutrénit, G. (2011). SMEs' absorptive capacities and large firms' knowledge spillovers: Micro evidence from Mexico. Paper no. 2011/0. Sweden: Centre for Innovation, Research and Competence in the Learning Economy (CIRCLE) Lund University.

De Fuentes, C. \& Dutrénit, G. (2007). The correlation between large firms 'knowledge spillovers and SMEs' absorptive capacities: Evidence for the maching industry in Mexico. Paper presentado en la MERIT Conference, United Nations University-Merit. Maastricht, Holanda.

Ferner, A. (1997). Country of origing effects and HRM in multinational companies. Human Resource Management Journal, 7(1), 19-37. 
Ferner, A. \& Quintanilla, J. (1998). Multinationals, national business systems y HRM: the enduring influence of national identity or a process of 'Anglo-Saxonización'. The International journal of Human Resource Management, 9(4), 710-731.

Ferner, A. \& Varul, M. (2000). "Vanguard” Subsidiaries and the Diffusion of New Practices: A Case Study of German Multinationals. British Journal of Industrial Relations, 38(1), 115-140.

Fosfuri, A., Motta, M. \& Rode, T. (2001). Foreing direct investment and spillovers throughtworkers'mobility. Journal of International Economics, (53), 205-222.

Gil, J. L. \& Solís, A. (2012). Derramas tecnológicas por la movilidad de empleados de multinacionales: Estrategias de capacitación de la Industria maquiladora de exportación. Interciencia, 37(9), 664-670.

Görg, H. (2007). Productivity spillovers from multinational companies. En Aylward, C. \& O’Toole, R. (Eds.). Perspectives on Irish Productivity (pp. 240-252). Dublín: Forfas.

Görg, H. \& Greenaway, D. (2001). Foreign direct investment and intra-industry spillovers: A review of the literature. Research Paper, (37).

Hofstede, G. (1980). Culture’s Consequences: International Differences in Work-Related Values. Beverly Hills: Sage.

Javorcik, B. (2004, junio). Does Foreign Direct Investment Increase the Productivity of Domestic Firms? In Search of Spillovers Through Backward Linkages. The American Economic Review, 94(39), 605-627.

Monge-González, R., Leiva, J. C. \& Alegre, J. (2011). Creación de empresas por parte de ex empleados de empresas multinacionales de alta tecnología en Costa Rica. Tec Empresarial, 5(3), 8-20.

Lévi-Strauss, C. (1966). The Savage Mind. Gran Bretaña: George Weidenfeld and Nicolson.

Lindholm, A. (1994). The economics of technology-related ownership changes. A study of innovativeness and growth through acquisitions and spin-offs. Suecia: Chalmers University of Technology.

Long, N. (2007). Sociología del desarrollo: una perspectiva centrada en el actor. México: CIESAS/COLSAN.

Parhankangas, A. \& Arenius, P. (2003). From a corporate venture to an independent company: a base for a taxonomy for corporate spin-off firms. Research Policy, 32(3), 463-481. 
Sethi, P. \& Elango, B. (1999). The influence of "country of origin" on multinational corporation global strategy: A conceptual framework. Journal of International Management, 5(4), 285-298.

Spencer, J. W. (2008). The Impact of Multinational Enterprise Strategy on Indigenous Enterprises: Horizontal Spillovers and Crowding out in Developing Countries. Academy of Management Review, 33(2), 341-361.

Tanev, S. (2012, marzo). Global from the Start: The Characteristics of Born-Global Firms in the Technology Sector. Technology Innovation Management Review, 2(3), 5-8. Recuperado de http://timreview.ca/article/532

Yan, Y. (2003). A comparative study of human resource management practices in international joint ventures: the impact of national origin. The International Journal of Human Resource Management, 14(4), 487-510. 